\section{M.K. Han}

University of Michigan Health System, Ann Arbor, MI, USA.

\section{REFERENCES}

1 Raghu G, Freudenberger TD, Yang S, et al. High prevalence of abnormal gastro-oesophageal reflux in idiopathic pulmonary fibrosis. Eur Respir J 2006; 27: 136-142.

2 Mays EE, Dubois JJ, Hamilton GB. Pulmonary fibrosis associated with tracheobronchial aspiration. A study of the frequency of hiatal hernia and gastroesophageal reflux in interstitial pulmonary fibrosis of obscure etiology. Chest 1976; 69: 512-515.

3 Tobin RW, Pope CE 2nd, Pellegrini CA, Emonda MJ, Sillery J, Raghu G. Increased prevalence of gastroesophageal reflux in patients with idiopathic pulmonary fibrosis. Am J Respir Crit Care Med 1998; 158: 1804-1808.

4 Patti MC, Tedesco P, Golden J, et al. Idiopathic pulmonary fibrosis: how often is it really idiopathic. J Gastrointest Surg 2005; 9: 1053-1056.

DOI: 10.1183/09031936.06.00071806

\section{From the authors:}

We appreciate M.K. Han's interest in the results of our study [1] and for the extended analyses of our data by calculating the risk for the population with acid gastro-oesophageal reflux (GER) and idiopathic pulmonary fibrosis (IPF). While this interesting observation deserves further study, as emphasised, it is apparent that the calculated risk is based on the number of GER disease $+=130 /$ number of cases + controls $=179 \times(\mathrm{OR}-1)$ / $\mathrm{OR}=.498$. Since an effective treatment regimen to improve patient outcome is yet to be determined for IPF, M.K. Han argues for patients with IPF to be treated for acid GER.

It is important to reiterate that our study design was not intended to investigate the causal effect of acid GER in IPF. While further studies are needed to clarify this in the pathogenesis of IPF, we have recently reported that some patients with IPF stabilise with adequate control of acid GER [2]. Only a double-blind, placebo-controlled, randomised clinical trial, with use of proton pump inhibitors (PPIs), can determine the efficacy of PPI in the treatment for IPF. In designing clinical trials using other agents, it may also be important to stratify randomisation based on the use of PPI at baseline and during the study period.

As more investigators and clinicians are recognising the high association of acid GER in IPF, M.K. Han's suggestion to treat IPF patients empirically with PPI will undoubtedly be raised again. We believe it is imperative to pursue further studies to determine the role of acid GER in the pathogenesis of IPF, and to determine if suppression of acid GER by medical and/or surgical treatment prevents further progression of IPF and/or improves outcome measures for patients with IPF.

We would like to thank M.K. Han for supporting our concepts by further analyses of our data. Hopefully, well-designed prospective clinical studies will be undertaken in the near future and the results will confirm the provocative observations.

\section{G. Raghu}

University of Washington, Seattle, WA, USA.

\section{REFERENCES}

1 Raghu G, Freudenberger TD, Yang S, et al. High prevalence of abnormal acid gastro-oesophageal reflux in idiopathic pulmonary fibrosis. Eur Respir J 2006; 27: 136-142.

2 Raghu G, Yang ST, Spada C, Hayes J, Pellegrini CA. Sole treatment of acid gastroesophageal reflux in idiopathic pulmonary fibrosis. Chest 2006; 129: 794-800.

DOI: $10.1183 / 09031936.06 .00067306$

\title{
Sandblasting jeans kills young people
}

\section{To the Editors:}

At this year's annual congress of the Turkish Thoracic Society (TTS), a mini-symposium was devoted to the occurrence of silicosis in the textile sector in Turkey. Clinicians from four cities reported on a total of 35 subjects with silicosis: 20, 10, four and one cases from Erzurum, Izmir, Tokat and Diyarbakir, respectively [1-4]. Nearly all patients were young males in their early twenties or even younger. They had been employed, often as undeclared workers, for only a few years in small workshops where denim jeans undergo jet sandblasting to give them a "worn-in" appearance. This job involved working long hours under very poor hygiene conditions, without any serious respiratory protection, and often far away from home, e.g. in Istanbul.

As no thorough occupational history had been taken or because silicosis was not suspected in these "textile" workers, several patients had initially been misdiagnosed as having tuberculosis (or sarcoidosis). So, depressingly beautiful images of high-resolution computed tomography scans or lung biopsies were projected, showing textbook presentations of acute silicosis (alveolar lipoproteinosis) or accelerated silicosis.

Two patients had started sandblasting when they were aged 13 and $14 \mathrm{yrs}$; both worked little more than 3 yrs and they died within a year of diagnosis, aged 18 and 19 yrs.

These two instances of silicosis in jeans sandblasters had already been reported at the 2005 congresses of the TTS and the European Respiratory Society, and they have now been published [5] (except for the fatal outcome). The suspicion of an epidemic now appears to be verified: an informal poll of $\sim 30$ participants in the mini-symposium at the TTS yielded another 15 cases from various other places in Turkey. 